\title{
Assurance en cas d'incapacité de gain
}

\section{Pour votre indépendance financière}

\author{
Votre problème \\ Vous avez perdu la vue par une grave maladie et vous \\ ne pouvez plus exercer votre profession. Les presta- \\ tions sociales obligatoires et votre prévoyance pro- \\ fessionnelle ne couvrent plus suffisamment votre \\ revenu.
}

Un accident de la circulation vous empêche pendant 18 mois d'exercer votre activité professionnelle. L'assurance-invalidité ne verse ses prestations qu'après un délai d'attente d'une année. Vous ne disposez donc d'aucun revenu pendant cette période.

Un accident de skis vous oblige de fermer votre cabinet pendant huit mois. En plus de votre manque à gagner, les coûts du cabinet et les salaires de vos assistantes sont à votre charge.

\section{Votre solution}

L'indemnité journalière d'invalidité complète les $1^{\mathrm{er}}$ et $2^{\mathrm{e}}$ piliers en cas d'une longue incapacité de gain jusqu'à l'âge de 65 ans
Prestations
Fr. 30.- à Fr. 250.- par jour
Délai d'attente 360 ou 720 jours

L'indemnité journalière de base apporte un remplacement du revenu pendant les deux premières années en cas d'incapacité de gain.
Prestations
Fr. 30.- à Fr. 600.- par jour
Délai d'attente
$20,30,60,90$ ou 180 jours

L'indemnité journalière complémentaire couvre les frais du cabinet pendant une année en cas d'incapacité de gain.

Prestations Fr. 30.- à Fr. 600.- par jour

Délai d'attente $20,30,60,90$ ou 180 jours

\section{Profitez de notre offre}

Vos besoins sont au centre de nos préoccupations. Notre nom vous garantit le conseil professionnel de nos conseillers qui possèdent une longue expérience dans le domaine des assurances, des produits bancaires et financiers. Grâce à notre indépendance nous pouvons vous offrir les meilleurs produits adaptés à vos besoins.

\section{Talon réponse}

Prénom / Nom

Adresse

NPA / Lieu

Date de naissance

Téléphone privé/cabinet

Atteignable le plus facilement (heure)

Adresse e-mail

J Je désire un conseil personnalisé. Veuillez $\mathbf{m}^{\prime}$ appeler.

Prière de $\mathbf{m}^{\prime}$ envoyer une offre personnelle.

Je $m^{\prime}$ intéresse aux thèmes suivants:
Caisse-maladie
○ $3^{\mathrm{e}}$ pilier $\mathrm{a}$ ou $\mathrm{b}$
Caisse de pension LPP
Responsabilité civile prof.
Placement de fonds

Roth Gygax \& Partner SA $₫$ Service de coordination Moosstrasse 2 - 3073 Gümligen

Téléphone 0319595000 — Fax 0319595010 mail@fmhinsurance.ch $\square$ www.fmhinsurance.ch 